\title{
Does Dexmedetomidine influence Somatosensory Evoked Potentials during spine surgery?
}

\author{
Asouhidou I, Zosimidis D, Giannaki Ch, Katsanevaki A, Charalampidis D, \\ Patsepas P. \\ Department of Anesthesia, G.Papanikolaou General Hospital, Thessaloniki, Greece.
}

\section{Background}

Spinal cord injury and postoperative neurologic deficit is a potent complication following posterior spinal fusion. The most commonly used monitor of nerve function during spinal surgery is the somatosensory evoked potentials (SSEP). Intravenous anesthetic agents as far as inhaled agents produce a dose depended increase in latency and decrease in amplitude of SSEP. Decreases in amplitude of $50 \%$ or more from the baseline associated with at least $10 \%$ prolongation in latency, is defined as clinically significant SSEP changes. As a result such changes should be immediately investigated. Dexmetedomidine-Dex is a highly selective $a_{2}$ adrenergic agonist, which is used as adjust in neuroanesthesia with intravenous or volatile agents. Dex not only minimizes the anesthetic agents but also the opioids, reducing the analgesic demands. What is already known is that Dex, in low dose does not affect SSEP. The current study aim to evaluate the effect of high dose of Dex on SSEPS in adults during posterior spinal fusion surgery.

\section{Material and methods}

Sixteen patients 18-75 years old, ASA I-III, scheduled for elective posterior spinal fusion surgery were enrolled in this prospective study. After induction in anesthesia, it was applied SSEP monitoring and a baseline test was performed after $15 \mathrm{~min}$ (in order to wash out the propofol used for induction). Infusion of Dexmedetomidine was started at a bolus dose of $1 \mathrm{mcg} / \mathrm{Kg}$ following by $0.7 \mathrm{mcg} / \mathrm{Kg}$. Bispectral Index (BIS) monitored the depth of anesthesia and an adequate level (40-50) of anesthesia was maintained by sevoflurane. SSEPs were recorded intraoperatively from the tibial nerve (P37) and data were analyzed over that period.

References

Penney R. Use of dexmedetomidine and ketamine infusions during scoliosis repair surgery with somatosensory and motor-evoked potential monitoring: a case report. AANA J. 2010;78(6):446-450.

\section{Results}

MAC of sevoflurane was stable through all the procedure with minor fluctuations (0.3-0.4 MAC). The amplitude was decreased $49.8 \%$ from the baseline (ampl bas:0.845 $\pm 0.433 \mu \mathrm{V}$, amplex: $0.424 \pm 0.193 \mu \mathrm{V}, \mathrm{p}=0.001)$. Latency presented $\mathrm{a}$ small prolongation with no clinical or statistical significant (latency bas: $45 \pm 9.27 \mathrm{msec}$, latency $y_{\text {dex: }}$ : $46 \pm 6.61 \mathrm{msec}, \mathrm{p}>0.05)$. None of the waves were lost after loading dose. All procedures were carried out without any surgical or anesthesiologic complications.
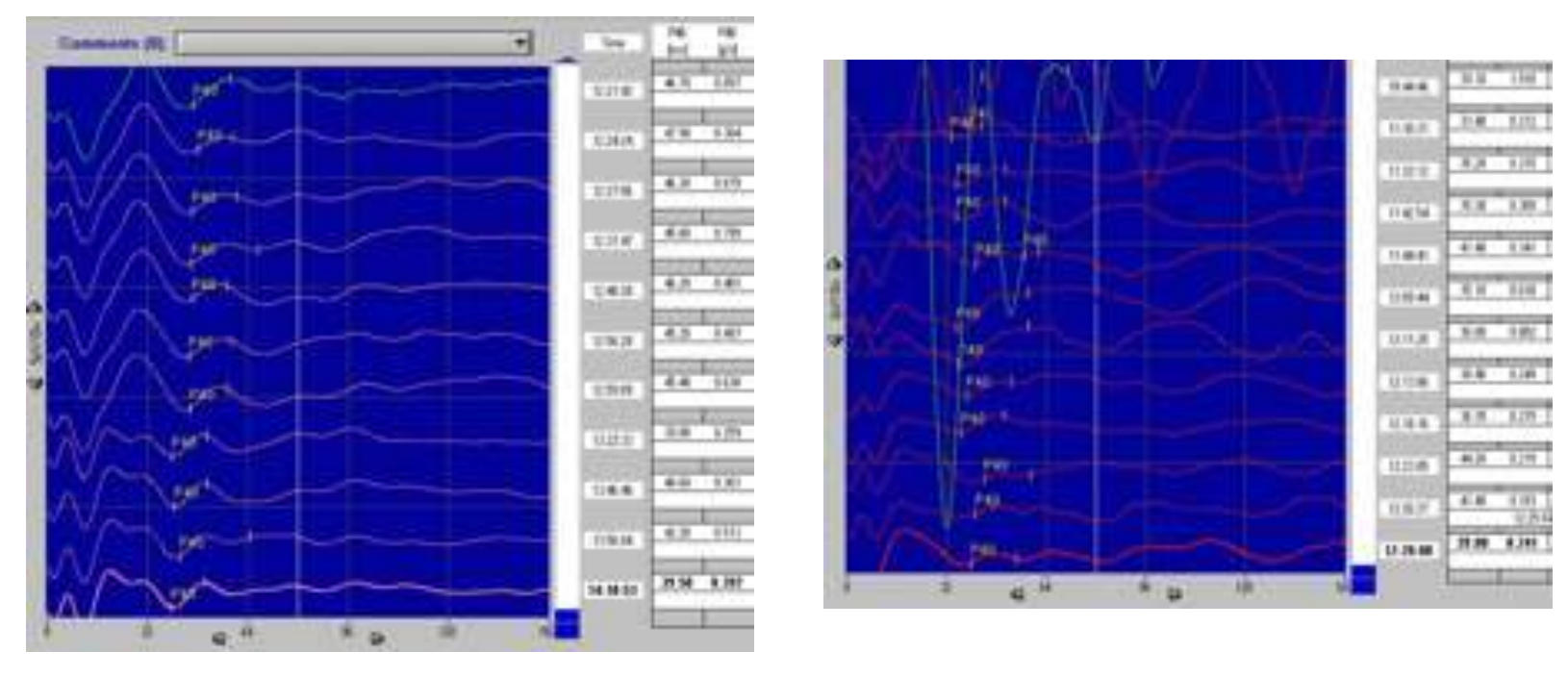

Fig.1 \&2. Intraoperative fluctuations of latency and amplitude of one patient. Amplitude is reduced significantly over time (from 0.857 to $0.397 \mu \mathrm{V}$ and 1.918 to $0.241 \mu \mathrm{V}$ ).

\section{Conclusion}

Dexmedetomidine even if administered in high dose of $1 \mathrm{mcg} / \mathrm{Kg}$ following of $0.7 \mathrm{mcg} / \mathrm{Kg}$, induced severe suppression of the amplitude but a minor prolongation of the latency which however did not reach the criteria to be clinical significant. Dexmedetomidine seems to constitute a safe adjust to general anesthesia during SSEP monitoring performing. 